BEITRÄGE ZUR RÜBENFORSCHUNG

Nr. 6 

W I S E N S C H A F T L I C H E A B A N D L U N G E N

Nr. 54

Sektion Pflanzenbau, Pflanzenzüchtung und Pflanzenschutz

\section{BEITRÄGE ZUR RÜBENFORSCHUNG}

Nr. 6

Mit 9 Abbildungen und 6 Tabellen

SCHRIFTLEITUNG:

PROF. DR. DR. O. HEINISCH

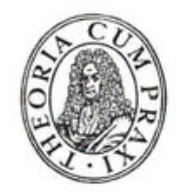

DEUTSCHE AKADEMIE

DER LANDWIRTSCHAFTSWISSENSCHAFTEN ZU BERLIN

AKADEMIE-VERLAG • BERLIN

1961 
Anschriften der Verfasser:

Dr. G. SENFF, Dr. P. CURTH

Institut für Pflanzenzüchtung Kleinwanzleben

der Deutschen Akademie der Landwirtschaftswissenschaften zu Berlin

Herausgegeben von der Deutschen Akademie der Landwirtschaftswissenschaften zu Berlin Die „Beiträge zur Rübenforschung“ erscheinen zweimal jähriich

Erschienen im Akademie-Verlag GmbH, Berlin W 8, Leipziger Straße 3-4

Copyright 1961 by Akademie-Verlag GmbH

Lizenznummer: $202 \cdot 100 / 442 / 61$

Kartengenehmigung: MdI der DDR Nr. 6531

Gesamtherstellung: VEB Druckerei „Thomas Müntzer“s Bad Langensalza

Bestellnummer: $2068 / 54 \cdot$ ES 22 D - Preis: D AI 17,-

Printed in Germany 